\title{
Interculturalidad e inclusión en Educación Física: innovación educativa Erasmus in Schools Interculturality and inclusion in Physical Education: educative innovation Erasmus in Schools Samuel López-Carril, Miguel Villamón, VicenteAñó \\ Universidad de Valencia(España)
}

\begin{abstract}
Resumen. Se describe la propuesta de innovación educativa Erasmus in Schools realizada en las clases de Educación Física y Lengua Extranjera: Inglés. a través de una unidad didáctica de 12 sesiones para dos grupos de $4^{\circ}$ de la ESO. El objetivo principal de la innovación, fue mejorar la convivencia escolar en las aulas y lograr la inclusión del alumnado inmigrado. Para ello, la innovación educativa siguió la tipología de distribución del alumnado conocida como ‘inclusion’, que incorpora la participación de otros miembros de la comunidad educativa en las clases. Así, estudiantes con beca Erasmus+ y voluntarios de la asociación Erasmus Student Network, compartieron su diversidad y experiencias con los estudiantes locales, incorporándose durante las clases al equipo docente. Los resultados indican un aumento de la participación y motivación de todo el alumnado en las clases de EF. Se percibió una disminución de los conflictos relacionados con el origen étnico y el género de los alumnos. Los erasmus que participaron en esta experiencia, se sintieron más incluidos en la sociedad de acogida. Se concluye que la puesta en práctica de la innovación educativa Erasmus in Schools, favorece la inclusión del alumnado inmigrado en las clases de EF.
\end{abstract}

Palabras clave: Educación inclusiva, Erasmus, Educación Física, Educación intercultural, Innovación educativa, Alumnado extranjero e Inmigración.

Abstract. It is described the educative innovation proposal Erasmus in Schools, which took place during the Physical Education and Foreign Language: English, lessons, through a didactic unit of 12 sessions for two groups of 4rth grade of a Spanish Secondary School. The main goal was to improve the coexistence and to achieve the inclusion of the foreign students. To do so, the educative innovation was based on a type of distribution named 'inclusion', which includes the participation of other members of the educative community in the classes. Thus, students with the Erasmus+ scholarship and volunteers of the Erasmus Student Network association shared their cultural diversity and experiences with the local students, involving themselves to the teaching team during the lessons. The results show a growth of the participation and motivation of all the students during the Physical Education lessons. Furthermore, it was perceived a decrease of the conflicts related with the ethnic origin and gender of the students. The Erasmus students that participated in this experience felt themselves more included into the host society. It is concluded that the implementation of the educative innovation Erasmus in Schools fosters the inclusion of the foreign students during the Physical Education classes.

Key words: Inclusive education, Erasmus, Physical Education, Intercultural education, Education innovation, Foreign students and Immigration.

\section{Introducción}

La inmigración es uno de los fenómenos sociales con más presencia mediática en la actualidad, que presenta retos tanto a nivel cuantitativo como cualitativo en áreas heterogéneas como: educación, política, economía, sanidad, entre otras. Este incremento de población extranjera en determinados lugares en períodos cortos de tiempo, genera situaciones que, si no son gestionadas de forma adecuada, pueden derivar en casos de racismo, segregación y discriminación (Ortiz, 2014).

La llegada de alumnado inmigrado durante las últimas décadas a los centros educativos españoles, es una realidad que está repercutiendo de forma importante en el sistema educativo (García, Rubio \& Bouachra, 2008). Así, la escuela se convierte en uno de los lugares donde la diversidad cultural es más visible, siendo muy importante que toda la comunidad educativa esté preparada y contribuya desde sus distintos ámbitos de actuación y posibilidades, a que se aproveche la diversidad cultural como una oportunidad de enseñanza-aprendizaje y no sea un obstáculo para la enseñanza que genere situaciones de no inclusión.

De esta forma, la población inmigrada en la escuela plantea a la comunidad educativa una serie de desafíos estructurales, organizativos, curriculares, etc., que, si no se afrontan desde una perspectiva inclusiva, ponen en riesgo la posibilidad de proporcionar una educación para todos de calidad. Con esta situación, cobra más relevancia si cabe el papel de la educación inclusiva como elemento clave para que todo el alumnado pueda aprender en igualdad de condiciones (HildebrandtStramann, 2016). Un tipo de enseñanza que haga posible conocer y reconocer esa nueva realidad desde el respeto, la tolerancia y la convivencia democrática (Fernández, 2003). Por su parte, autores como Flores, Prat y Soler (2014) señalan que, ante la vigencia actual del fenómeno de la inmigración y su importancia en el ámbito escolar, conviene que los docentes reciban una formación específica sobre este fenómeno social, para que puedan establecer las acciones educativas más adecuadas a cada contexto con tal de lograr la inclusión de todos los alumnos, provengan de donde provengan.

Fecha recepción: 14-06-17. Fecha de aceptación: 21-12-17 Samuel López-Carril samuel.lopez@uv.es
Con el fin de lograr una escuela más inclusiva, emerge la educación intercultural como una de las posibles corrientes pedagógicas que se puede trabajar en las distintas áreas de conocimiento de la escuela. Sáez (2006), expone que a los ciudadanos de la sociedad multicultural propia del siglo XXI les conviene desarrollar una educación intercultural que vaya más allá de los muros de las escuelas. Ello permitirá respetar, comprender y valorar más la diversidad en general, y la derivada de la inmigración en particular, transformando el miedo a lo desconocido, a poder vivir una oportunidad de enriquecimiento cultural, personal y social. Además, dicho autor insiste en que esa riqueza cultural, que los inmigrantes pueden aportar a la sociedad, ayuda a incrementar las formas en las que los ciudadanos perciben y resuelven los problemas personales y sociales. De esta forma, la educación intercultural da la oportunidad de adquirir una visión más amplia y completa del ser humano, ayudando a hacer frente a problemas de gran calado social como el racismo o la intolerancia.

En la línea de lo anterior, Aguado (1996) define la educación intercultural como aquella basada en la diferencia, la diversidad y la pluralidad cultural, más allá de ofrecer una educación específica para los que son culturalmente distintos. Es una educación dirigida y destinada a toda la población. Se trata de proporcionar a los alumnos un modelo educativo holístico e integrador que favorezca la igualdad de oportunidades y resultados, superando discriminaciones y conductas racistas. Como objetivo central, la educación intercultural busca que cada persona sea capaz de desarrollar de forma crítica su identidad, a la vez que aprecia la del resto de personas a través de la interacción. En síntesis, la educación intercultural aboga por el reconocimiento y respeto hacia la diversidad, con tal de crear una sociedad más democrática basada en la igualdad, la tolerancia y la solidaridad, donde el diálogo y el espíritu crítico entre los ciudadanos, ayude a crear un marco de respeto y reconocimiento hacia la diversidad y todo lo que ésta nos enriquece.

Por su parte, autores como García-Fernández y Goenechea (2009) apuntan a una visión de la educación intercultural como una tautología, donde no sea necesario aplicar nuevos modelos pedagógicos, ya que se parte de la premisa de que toda acción educativa debe atender per se la diversidad del alumnado. Así, Flores, Prat y Soler (2015b) inciden en que más allá del discurso teórico sobre la educación intercultural, hay que atender la diversidad cultural de forma específica en las aulas desde 
el análisis y la comprensión de cada realidad, para tratar de establecer las actuaciones educativas oportunas para cada contexto educativo.

\section{La interculturalidad en la Educación Física (EF)}

Para Soler, Flores y Prat (2012), las dos instituciones que tienen un papel clave a la hora de fomentar la inclusión de la población inmigrante, por su cercanía a la ciudadanía, son la escuela y la administración pública. Es por ello que la escuela, tal y como se ha ido exponiendo anteriormente, es un ámbito muy importante para lograr la inclusión de todas las personas en la sociedad, siendo la EF la asignatura donde se puede trabajar la inclusión con grandes posibilidades de éxito, gracias a todos los aspectos educativos únicos que ofrece la educación a través del movimiento.

Por otra parte, la actividad física y deportiva es una de las manifestaciones sociales con mayores posibilidades de ser aprovechadas para trabajar la educación intercultural (Maza, 2002). Según Medina (2002) el deporte es un elemento definidor de la identidad que es susceptible de ser utilizado para la inclusión de personas de distintas culturas. Sin embargo, autores como Carter-Thuillier, López-Pastor y Fuentes (2017), resaltan la dimensión pedagógica que la EF posee y que no se da en ocasiones en el contexto del ámbito deportivo.

Según Flores (2013), la EF posee una serie de particularidades interesantes y ventajosas para trabajar la interculturalidad, en comparación con otras áreas curriculares. Así, encontramos en las clases de EF una mayor implicación psicosocial del alumnado, fruto de las numerosas oportunidades de interacción social entre ellos y el profesorado que proporciona la comunicación corporal. Además, estas interacciones pueden favorecer la creación de relaciones interpersonales, lo que ayuda a comprender y a conocer más al compañero. Flores (2013) prosigue defendiendo el alto componente motivacional que tiene la $\mathrm{EF}$, algo que puede fomentar una mayor implicación activa, favoreciendo el aprendizaje de una serie de valores, creencias y actitudes más abiertas y respetuosas con la realidad multicultural del alumnado.

En la misma línea, López-Pastor, Pérez y Monjas (2007) señalan como otro aspecto importante, que en las clases de EF se trabaje mediante el lenguaje universal de la comunicación motriz y el movimiento. De esta forma se superan las posibles barreras lingüísticas que impiden la comunicación verbal entre los alumnos y el profesorado en otras materias, al no ser un requisito indispensable dominar la lengua vehicular en las clases de EF. Las características específicas de la EF expuestas durante el desarrollo de este apartado del trabajo, han sido valoradas positivamente por autores como como Flecha y Puigvert (2002, 2011), como elementos didácticos favorecedores para el desarrollo de la educación intercultural en las clases deEF desde una perspectiva comunicativa, crítica y transformadora.

Por su parte, autores como Derri, Kellis, Vernadakis, Albanidis y Kioumourtzoglou (2014) señalan en las conclusiones de su estudio la necesidad de incluir elementos vinculados con la educación intercultural en el currículum de EF con tal de intentar cambiar los estereotipos y visiones perjudiciales respecto de los estudiantes respecto a los inmigrantes. En el contexto español, otros autores como Soler et al. (2012), afirman que la EF puede contribuir de forma especial al desarrollo de la interculturalidad a través de dos de las competencias recogidas en su currículum. La primera de ellas es la competencia social y ciudadana, en donde a través de la práctica física y las relaciones interpersonales se puede aprender una serie de valores como el respeto hacia las diferencias. En segundo lugar, tal y como se ha especificado anteriormente, desde la EF se puede trabajar la competencia comunicativa, que en este caso se realiza sobre todo a partir de experimentar con el cuerpo y el movimiento como forma de expresión y comunicación.

Por último, Flores, Prat y Soler (2017), en un estudio donde analizaron las percepciones del profesorado de EF sobre los efectos y consecuencias que ha generado y/o genera la presencia de alumnado extranjero en las aulas, concluyen que, en general, para la mayoría docentes de EF (83\%) no hay efectos negativos en el contexto de las clases de EF, aunque sí perciben efectos muy negativos en el aula ordinaria, añadien- do el 66\% de ellos, que desde una perspectiva global, la inmigración en las escuelas es un problema para el que los docentes no disponen de recursos ni preparación. Además, más allá de todos los aspectos positivos que tiene la EF a la hora de dar una respuesta educativa a la diversidad cultural, cabe resaltar que en sí misma, la EF no es inclusiva, sino que la inclusión del alumnado depende realmente de la actuación de los profesionales docentes. De hecho, en otro estudio de estos mismos autores (Flores, Prat \& Soler, 2015a), se detecta que algunos docentes deEF perciben el alto porcentaje de alumnado de origen extranjero en las aulas como un elemento que puede desgastar al profesorado, llevándoles en algunas ocasiones a la búsqueda de cambio de centro. Siendo conocedores de esta realidad, es importante que se forme correctamente a todo el equipo docente para que EF pueda convertirse en una herramienta que permita trabajar la inclusión educativa en el contexto de una sociedad multicultural.

\section{Desafíos para una EF realmente inclusiva}

A pesar de las oportunidades específicas que ofrece la EF para promover la interculturalidad, según Maza (2002), no siempre se ha trabajado la inclusión del alumnado de forma adecuada. En algunas ocasiones se tiende a perpetuar en las clases de EF los modelos estereotipados que predominan en la sociedad. Maza (2002) lo justifica basándose en la tendencia de los centros educativos a realizar fiestas o jornadas multiculturales en las que se difunden las distintas culturas, pero categorizándolas de forma inconsciente, al pasar a ser meras exposiciones temporales. Por ejemplo, en el caso de la EF, en ocasiones los docentes recurren a desarrollar unidades didácticas sobre danzas, músicas y deportes de otras culturas. Sin embargo, éstas se desarrollan de forma superficial, con la idea de dar a conocer o difundir otras posibilidades de movimiento de distintos contextos culturales. Esto en sí no es negativo, ya que conocer es el primer paso hacia la interculturalidad, pero no es suficiente si todo se queda en una exposición categorizada y no se profundiza más allá de los estereotipos, que son los que se suelen compartir en nuestra sociedad.

En la misma línea argumental, Flores, Prat y Soler (2015b) insisten en que no es suficiente con la mera práctica de un juego, danza, o deporte típico de un país en concreto, para hacer sentir al alumnado recién llegado su inclusión entre el resto de compañeros. Por lo tanto, se debe profundizar más sobre cómo se trabaja la interculturalidad dentro del currículum de la EF, su continuidad a lo largo del curso académico, y la transversalidad con otras materias ya que los resultados serán más positivos. La propuesta de innovación educativa Erasmus in Schools que se expone en este artículo, busca incidir sobre esta posible superficialidad a la hora de trabajar la interculturalidad en las clases de EF, profundizando en el intercambio cultural en una experiencia educativa interdisciplinar, compartida tanto por los alumnos como por otros agentes sociales.

\section{Contextualización de la innovación educativa Erasmus in Schools}

La educación intercultural, tal y como se ha expuesto anteriormente, busca terminar con las desigualdades y los procesos de segregación del alumnado. Problemáticas, que en ocasiones son causadas por decisiones tomadas a nivel político en materia de educación, o por la propia comunidad educativa. Una de las posibles formas de buscar soluciones a estas situaciones de desigualdad, pasa por desarrollar alguna innovación educativa. Concepto que, parafraseando a Carbonell (2006), queda descrito como aquel proceso nutrido de un conjunto de ideas, estrategias, con una mayor o menor sistematización, que son llevados a cabo con la idea de introducir y provocar cambios en las prácticas educativas vigentes. Para ello se modifican actitudes, concepciones, intervenciones, tratando de transformar y mejora los procesos de enseñanza y aprendizaje. En definitiva, se trata de producir un cambio educativo y un impacto en la comunidad escolar. Por su parte, Imbernón (1996) afirma que: 
«la innovación educativa es la actitud y el proceso de indagación de nuevas ideas, propuestas y aportaciones, efectuadas de manera colectiva, para la solución de situaciones problemáticas de la práctica, lo que comportará un cambio en los contextos y en la práctica institucional de la educación» (p.64).

Siguiendo con el espíritu descrito por los dos autores anteriores, la innovación educativa Erasmus in Schools que se presenta en este artículo, nace como respuesta a la detección por parte del profesorado de EF de problemas de convivencia y de exclusión, entre el alumnado de un instituto del barrio de Benimaclet (Valencia), causadas a priori, por la diferenciación del alumnado en dos grupos independientes, configurados en función de la elección por parte del alumnado de la lengua vehicular de enseñanza. Así, la intención del desarrollo de la misma es producir cambios en la actitud, ideas, comportamiento, etc., tanto por parte del alumnado como del profesorado, en relación a la inclusión educativa basada en la diversidad cultural, en el contexto de las clases de EF.

La distribución del alumnado en dos o más grupos distintos no es infrecuente dentro del contexto educativo español. Cuando dentro de un mismo centro escolar, estas divisiones se basan en la aplicación de criterios como la adaptación del currículum a los distintos alumnos en función de su rendimiento y diversidad, dividiendo así al alumnado en grupos homogéneos en función de sus capacidades/aptitudes, se aplica una organización del alumnado conocida como streaming (Comisión Europea, 2006). En el sistema educativo español, la mayoría de centros siguen este tipo de distribución del alumnado, después de que la LOGSE fomentara el desdoble de grupos para atender las necesidades educativas específicas. Este tipo de organización busca dar una respuesta al fracaso de la organización mixture, pudiéndose atender así la diversidad de una forma especializada en grupos homogéneos (De Botton, Flecha \& Puigvert, 2009)

En el contexto de la aplicación de la innovación educativa Erasmus in Schools, la agrupación del alumnado siguiendo el modelo streaming, se produce en el momento de la escolarización a través de la elección previa por parte del alumnado de la línea de enseñanza en castellano o la de valenciano. En función de esta decisión, se configuran dos grupos diferenciados que se mantienen independientes uno del otro a lo largo de toda la escolarización.

El idioma es uno de los primeros grandes retos que deben afrontar los inmigrados al llegar a su nuevo centro de estudios. En el instituto donde se aplicó la innovación educativa Erasmus in Schools, la línea en valenciano se convierte en una barrera lingüística para el alumnado recién inmigrado, que, en muchas ocasiones, ya de inicio desconoce el castellano. Esto provoca que la mayoría de ellos eviten la línea en valenciano creándose así, al aplicar el modelo streaming, dos grupos con características muy distintas. Ejemplo de ello es la distribución del alumnado que participó en la innovación educativa repartido en dos grupos: $4^{\circ}$ de ESO «A», de línea en castellano, compuesto por 30 alumnos, de los cuales únicamente tres de ellos eran de nacionalidad española, mientras que el grupo de $4^{\circ}$ de ESO «B», de línea en valenciano, estaba formado por un total de 27 alumnos, de los cuales únicamente cuatro eran de nacionalidad no española. Estas diferencias cuantitativas visibles, en cuanto a la procedencia del alumnado, reflejan una desemejanza palpable entre ambos grupos, apareciendo un mayor número de problemas de convivencia, de rendimiento escolar, etc., en el de línea en castellano que en el de línea en valenciano.

En este contexto, tras reflexionar sobre la dinámica de las clases en las que se observaron conductas y actitudes entre los alumnos de rechazo cultural, una falta de interacción entre los chicos y las chicas y un peor rendimiento académico por parte del grupo en castellano respecto al de valenciano, se decidióllevar a cabo la innovación educativa Erasmus in Shools, para tratar de romper esta dinámica escolar y lograr un cambio basado en la inclusión educativa. Para ello, se diseñó una unidad didáctica (UD) en la que se intentó transformar el modelo de agrupación streaming, configurado basándose en la elección de la línea vehicular de enseñanza, a una organización del alumnado que siguiese los principios del modelo inclusión (Duque, Holland \& Rodríguez, 2012).
La organización del alumnado conocida como inclusion englobauna serie de actuaciones educativas que buscan dar una respuesta positiva a la diversidad del alumnado, manteniendo la heterogeneidad en las aulas e incorporando en su interior recursos para poder atender dicha diversidad y crear oportunidades de enseñanza-aprendizaje más inclusivas (Cifuentes, Siles \& Torrego, 2012).

Planteamiento didáctico de la innovación educativa Erasmus in Schools

La propuesta educativa Erasmus in Schools consistió en la realización de una UD de deportes, actividad física y juegos populares del mundo, que se realizó durante cuatro semanas con dos grupos de $4^{\circ} \mathrm{de}$ ESO de un instituto del barrio de Benimaclet en Valencia. La UD se caracterizó por la presencia durante las clases de estudiantes internacionales quecursaban unabeca Erasmus+, así como alumnos dela Universitat de València, pertenecientes a la asociación Erasmus Student Network (ESN).

Un total de 10 estudiantes erasmus y siete voluntarios de ESN participaron de forma activa en las sesiones durante el desarrollo de la innovación. Cada estudiante internacional acudió al centro escolar durante una semana, en función de su nacionalidad (italiana, alemana, estadounidense y francesa), mientras que los voluntarios de ESN acudieron sin seguir ningún patrón en concreto, a modo de apoyo y de enlace entre el equipo docente y los estudiantes internacionales.

La intervención de los estudiantes internacionales se iniciaba los lunes en una clase teórica de Lengua Extranjera: Inglés (LEI), donde introducían al alumnado aspectos culturales de su lugar de origen y contenido vinculado a la actividad física y deportiva de cada país, mientras que los martes y jueves acudían a las dos clases prácticas semanales de EF de 55 minutos de duración, participando de forma activa en el desarrollo de las sesiones tal y como queda expuesto en la Tabla 1.

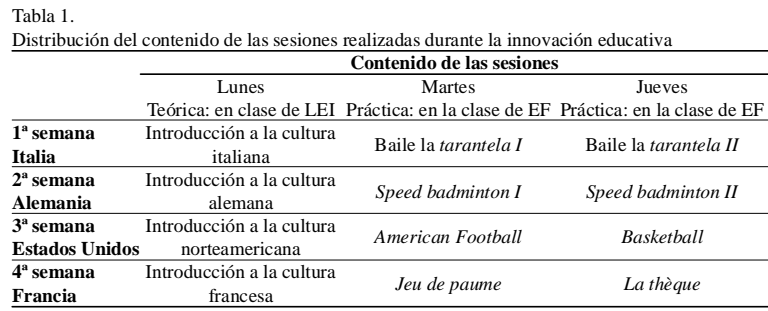

En el caso de las clases de LEI se trabajaron los objetivos y contenidos de la UD Erasmus in Schools de forma transversal. Por un lado, esta sesión servía a modo de presentación y familiarización de los erasmus que iban a venir a las sesiones de EF durante el resto de la semana. Por el otro, se trabajaron en lengua inglesa, los aspectos geográficos, culturales, etc., propios del lugar de origen de cada estudiante internacional, así como se explicaba de forma teórica el contenido, objetivos y desarrollo de las actividades a realizar posteriormente en las clases prácticas de EF.

Tanto el contenido de las clases teóricas en la signatura de LEI como el de las prácticas de EF fue trabajado y consensuado previamente entre el equipo docente, los estudiantes internacionales y los voluntarios de ESN, con intención de involucrarles de forma directa en el proceso de enseñanza-aprendizaje y convertirles durante el desarrollo de las sesiones en parte del equipo educador. De esta forma, se siguió la filosofía del modelo de organización del alumnado inclusion (Cifuentes, Torrego \& Siles 2012), incrementando la presencia de adultos en el aula para mejorar la atención de la diversidad del alumnado

\section{Acceso a los estudiantes erasmus}

Para acceder a los estudiantes erasmus se recurrió a la asociación sin ánimo de lucro ESN, cuyo objetivo principal es apoyar todo aquello vinculado a la movilidad internacional, ayudando a los estudiantes que 
cada año deciden estudiar un programa de intercambio en el extranjero. ESN recibe el apoyo de la Comisión Europea y está presente en 40 países de toda Europa y en más de 500 universidades, entre ellas la Universitat de València.

Los voluntarios de ESN facilitaron el contacto con erasmus, compartiéndoles el proyecto de esta UD, para que acudiesen a las clases de EF y enseñasen a los alumnos deportes, actividades y juegos típicos de su cultura. En cuanto a la decisión sobre la procedencia geográfica de los erasmus que participaron en la UD, se intentó que hubiese el mayor grado de diversidad cultural posible, basándose en la disponibilidad de los erasmus que se prestaron voluntarios. Una vez confirmados los estudiantes internacionales que querían participar en la experiencia, se decidió que cada semana de la UD estuviese dedicada a desarrollar el contenido específico de una cultura, asistiendo a las clases durante dicho período grupos de estudiantes de la misma nacionalidad.

\section{Objetivos, contenido, organización y temporalización de las sesiones de la UD}

Tal y como se ha comentado anteriormente, todas las sesiones se prepararon en reuniones que se realizaron previamente antes del inicio de la UD, en la que estuvieron presentes los voluntarios de ESN y los estudiantes erasmus. En éstas se determinaron los aspectos más relevantes a la hora de preparar las sesiones de la UD: los objetivos, el contenido, la organización y papel que iba a tomar cada educador en cada momento de las sesiones, la estructura de las distintas actividades y su temporalización (ver Tabla 1).

En relación a los objetivos, estos configuraron el tipo de propuesta planteada en la UD Erasmus in Schools, buscando dar respuesta a la situación del centro descrita en el apartado de contextualización de este trabajo. Así, la innovación educativa persiguió durante el desarrollo de las sesiones los siguientes diez objetivos:

1. Reflexionar sobre aspectos ideológicos en el profesorado y el alumnado respecto a la diversidad cultural, donde predominan estereotipos culturales.

2. Desarrollar en el alumnado, en los erasmus y en los voluntarios de ESN un interés por comprender mejor las distintas culturas, valorando la diversidad como un elemento que enriquece a la sociedad.

3. Incluir a los estudiantes extranjeros tanto en las aulas como en la sociedad que los acoge a través de una propuesta de UD inclusiva.

4. Trabajar de forma transversal la educación intercultural en un centro educativo, implementando una propuesta de UD desarrollada en más de una asignatura.

5. Abrir la escuela a su entorno, para que intervengan en el proceso de enseñanza-aprendizaje otros miembros de la comunidad educativa.

6. Aprovechar el potencial que tiene la EF para trabajar la educación intercultural a través del movimiento.

7. Fomentar el aprendizaje instrumental y emocional a través de la agrupación del alumnado en el aula conocida como inclusion.

8. Aumentar el grado de conocimiento de la actividad física y deporte procedente de otros lugares del mundo.

9. Mejorar la comunicación lingüística del idioma inglés.

10. Reflexionar sobre los estereotipos construidos alrededor de los estudiantes de las becas Erasmus y ayudar a cambiar esta visión tan superficial de los mismos.

En cuanto al contenido trabajado durante el desarrollo de la UD (ver Tabla 1), este se puede ubicar dentro de los siguientes bloques del currículo LOMCE del área de EF en ESO y $1^{\circ}$ de Bachillerato: Bloque 2: Juegos y deportes, Bloque 4: Expresión Corporal y Bloque 5: Elementos transversales a la asignatura. Así, fue durante las reuniones previas con los estudiantes internacionales, cuando se iban decidiendo el contenido a realizar, con base a los tres bloques temáticos indicados, a los propios conocimientos que los estudiantes internacionales tenían respecto a la EF y el vínculo de las propuestas con los objetivos de la UD anteriormente expuestos.

Además, más allá de los contenidos tratados, a lo largo del desarro- llo de la UD se puso énfasis en trabajar las siguientes tres competencias básicas:

«Competencia lingüística»: desarrollándose en el alumnado tanto la capacidad de interacción con otros individuos en el lenguaje del movimiento y en la lengua extranjera inglesa.

«Competencia de conciencia y expresiones culturales»: trabajando propuestas prácticas proveniente de otras culturas incidiendo en la reflexión crítica durante su realización y a la conclusión de las sesiones, desarrollando una actitud abierta y de respeto hacia las mismas.

«Competencias sociales y cívicas»: centrándonos en los procesos y las interacciones entre el alumnado, buscando un intercambio cultural entre los estudiantes erasmus, los voluntarios de la asociación ESN y el alumnado, respetando la diversidad cultural y valorando las diferencias como un aspecto positivo que favorece la convivencia ciudadana.

Por otra parte, siguiendo a Martos (2009), ubicamos la innovación Erasmus in Schools en el área de EF inclusiva, ya que la innovación busca la total participación del alumnado con las máximas garantías, persiguiendo la inclusión del alumnado inmigrante en la sociedad, siendo la escuela un lugar de actuación muy relevantea la hora de alcanzar dicho objetivo. Así, el planteamiento de la innovación educativa Erasmus in Schools, puso especial atención en el proceso y no únicamente en el resultado. Tuvo un carácter flexible y abierto, contextualizado a la realidad del centro, abriéndose así hacia la realidad más inmediata, en este caso, en el barrio de Benimaclet. Durante el desarrollo de la UD, se establecieron conexiones con elementos del mismo, como, por ejemplo, los estudiantes erasmus que residen en el mismo barrio y los voluntarios de ESN, para proponer situaciones de enseñanza/aprendizaje lo más variadas y significativas posibles.

\section{Resultados más relevantes y reflexión sobre lo ocurrido}

A lo largo del desarrollo de la innovación educativa Erasmus in Schools, desde el equipo docente se percibió un impacto positivo en la dinámica de las clases de EF, en los dos grupos de $4^{\circ}$ deESO, mejorando la convivencia del alumnado y su participación en las clases, sobre todo en el grupo de $4^{\circ}$ de ESO «A», compuesto casi en su totalidad por estudiantes inmigrados, algo que el equipo docente apreció durante el desarrollo de las sesiones, en comparación con las dinámicas de trabajo que venían desarrollándose hasta el momento.

En parte, se entiende que los resultados positivos expuestos pueden ser debidos a las indicaciones de Fernández (2001), quien defiende que la educación intercultural se debe desarrollar siguiendo tres premisas de trabajo: flexibilidad interna (la metodología seguida fue muy flexible adaptándose constantemente a las distintas realidades de lo que ocurría en las sesiones), apertura al entorno (en un mes intervinieron en las clases deEF personas externas a la escuela como los estudiantes erasmus y los voluntarios de ESN) y responsabilidad profesional (dada la realidad observada en clase durante los primeros días se buscó una solución a los problemas derivados del fenómeno de la inmigración y de la organización del alumnado, de tipo streaming, para diseñary llevar a cabo la presente innovación educativa).

Por otro lado, durante el desarrollo de las sesiones se identificaron menos conductas irrespetuosas hacia los compañeros por motivos de origen étnico, respecto a las que se daban antes de realizar la innovación educativa. Además, se dieron menos conflictos derivados de diferencias de género (menos discusiones y menor oposición a realizar actividades en parejas). Estos aspectos mejoraron la dinámica de las clases, disminuyendo el número de interrupciones que habitualmente se venían dando.

Además, en relación con los resultados de la innovación respecto a los estudiantes erasmus, su satisfacción con la experiencia fue muy positiva, según se desprende de sus propias palabras recogidas en un formulario de retroalimentación que realizaron tras finalizar su intervención cada semana, asegurando todos que repetirían la experiencia si se les diese la oportunidad.

Por otra parte, se percibió una mayor motivación general del 
alumnado hacia las clases de EF y LEI, reflejada en una mayor participación activa durante las sesiones y en la entrega de unas fichas a completar por el alumnado sobre las actividades realizadas semanalmente. Resultados que van en la línea de los expuesto por autores como Alonso, Cachón, Castro y Zagalaz (2015) quienes recomiendan diseñar y aplicar UUDD sobre juegos populares tradicionales ingleses, por su impacto positivo en la motivación e implicación del alumnado, a la vez que permite el aprendizaje de una lengua extranjera.

Los resultados compartidos refuerzan lo expuesto por Sáez (2006) respecto a las posibilidades que ofrece una escuela más abierta a su entorno para aprovechar todas las oportunidades de enseñanza-aprendizaje queéste ofrece. Deesta manera, la innovación Erasmus in Schools creó situaciones de interacción y diálogo defendidas por Bartolomé (2001) entre los alumnos locales y los erasmus, que contribuyeron a que los estudiantes de intercambio se sintiesen un poco más integrados en la sociedad que les acoge, teniendo una primera experiencia en el sistema educativo español no universitario en contacto con estudiantes locales. Por otra parte, los alumnos locales pudieron conocer qué es el programa de intercambio Erasmus+ y las oportunidades que éste ofrece. Gracias a esta experiencia interactiva, esta innovación educativa ayuda a reflexionar sobre los estereotipos que la sociedad española tiene con el colectivo erasmus al que se le suele vincular con la fiesta y el poco aprovechamiento académico.

\section{Limitaciones y problemas acontecidos}

Las principales dificultades estuvieron relacionadas con el gran reto de buscar a erasmus que estuviesen dispuestos, de forma voluntaria, a venir al centro educativo y compartir su tiempo libre con los alumnos. A la hora de preparar las sesiones, se requirió un trabajo constante y esfuerzo, sobre todo a nivel de comunicación, para poder coordinarse con ellos y seleccionar temas atractivos que se ajustasen a los objetivos de esta UD, de cara a trasladárselos a los alumnos. Además, no siempre fue sencillo fijar los encuentros previos a la visita a las escuelas con los erasmus y los voluntarios de ESN.

Otro problema ocurrido está relacionado con el idioma, ya que algunos erasmus únicamente podían hablar en inglés y no todos los estudiantes dominaban esa lengua. No obstante, con la ayuda de los voluntarios de ESN y las posibilidades de comunicación a través del movimiento que ofrece la $\mathrm{EF}$, esta barrera lingüística fue superada.

Por último, no podemos olvidar que esta innovación fue aplicada durante un mes y que no se realizó una investigación sistematizada para poder valorar científicamente el impacto real de la experiencia. Por ello, coincidiendo con lo expuesto en trabajos como el de Ruiz, Molero, Zagalaz y Cachón (2012) debemos ser cautos con la generalización de los resultados obtenidos en nuestro estudio, los cuales no deben ser extrapolados a otros contextos por las propias limitaciones de la experiencia. Así, sugerimos para futuros estudios, que a nivel metodológico se sigan los procesos adecuados para poder ofrecer a la comunidad académica y educativa unos resultados con validez científica que puedan ser generalizados. También sugerimos que esta experiencia se extienda más en el tiempo y que se aplique de forma transversal en más asignaturas o como proyecto educativo de centro, pudiendo valorar la permanencia y nivel de consolidación de los resultados descritos en el presente artículo.

\section{Discusión y conclusiones}

La inmigración continúa siendo una asignatura pendiente por resolver en el sistema educativo español. Así, resulta necesario plantear desde el sistema educativo una serie de principios y pautas generales que ayuden a lograr una verdadera inclusión intercultural para que, desde cada centro escolar, se establezcan actuaciones educativas específicas en función del contexto en el que se encuentra cada uno de ellos. Independientemente de lo anterior, ya sea desarrollando políticas educativas generales o a través de acciones educativas de centro, el enfoque educativo de la escuela en relación al fenómeno de la inmigración debe estar dirigido hacia la trasmisión de valores que favorezcan la inclusión y la construcción de una sociedad más justa y humana, mediante una educación intercultural (Fernández, 2001). La innovación educativa Erasmus in Schools, refuerza la importancia de diseñar este tipo de estrategias y propuestas inclusivas en el contexto de las clases de EF, brindando una oportunidad para contribuir a la inclusión del alumnado tanto en el ámbito educativo como en el social.

En relación a los resultados logrados con el aumento de las interacciones y participación del alumnado en las clases, estos van en sintonía de lo expuesto por Flores (2013) y López-Pastor, Pérez y Monjas (2007), quienes indican que la EF ofrece la ventaja, frente a otras materias, de trabajar la interculturalidad gracias al lenguaje universal de la comunicación motriz y el movimiento, superando así barreras lingüísticas como lo pueda ser el no dominio de la lengua vehicular de enseñanza. En las clases se ha podido observar como prácticamente todo el alumnado trabaja de forma activa, independientemente de la nacionalidad y el género, algo que refuerza lo expuesto por Soler et al. (2012) quienes señalan que la EF es la asignatura más adecuada para aprender una serie de valores, como el respeto a las diferencias a través de las relaciones interpersonales, que se crean en las sesiones prácticas.

Por otra parte, según la experiencia desarrollada, el modelo organizativo inclusion, favorece un mayor desarrollo personal y autoestima en los alumnos, frente al streaming y el mixture, sobre todo entre los estudiantes de origen extranjero. Además, trabajar la interculturalidad siguiendo el modelo inclusion en las clases de EF, da la oportunidad a que el alumnado pueda comunicarse mediante el movimiento, evitando barreras lingüísticas y recibiendo una mayor atención docente. Igualmente, a mayor número de recursos humanos en las clases de EF, más oportunidades se tendrá para observar todo lo que acontece en las mimas, para posteriormente reflexionar sobre ello y proponer cambios de cara a mejorar la práctica docente.

Respecto a la presencia de estudiantes erasmus en las clases de EF, podemos afirmar que la innovación educativa Erasmus in Schools, ha sido una experiencia muy positiva tanto para los alumnos, los estudiantes de intercambio, los voluntarios de ESN y el equipo docente. Los erasmus, después de haber participado en esta experiencia, según la retroalimentación facilitada, afirman que conocen mejor la cultura española y el sistema educativo español. Por su parte, los estudiantes locales tras la vivencia de esta experiencia, manifestaron su deseo de disfrutar de una beca Erasmus+ en el futuro.

Esta innovación puede considerarse como un ejemplo de propuesta educativa inclusiva realizada desde un centro escolar, que posibilita la mejora la dinámica general de las clases, tal y como apuntan los estudios de Brown (2001), De Botton etal. (2009) y Ragvid(2005). Después de vivenciar esta experiencia, reforzamos la convicción de que la educación intercultural favorece la inclusión de todo el alumnado mejorando la convivencia en las aulas. Además, cuanta más diversidad haya en el aula, sea del tipo que sea, mayores serán las oportunidades de aprendizaje y de enriquecimiento personal, ofreciendo mayores oportunidades de enseñanza-aprendizaje tanto al alumnado como al equipo docente y el conjunto de la comunidad educativa.

La educación intercultural inclusiva es cada día más necesaria en el contexto de sociedad multicultural en la que vivimos, siendo la EF una asignatura con oportunidades extraordinarias frente a otras materias, para trabajar la interculturalidad en sus sesiones. Autores como CarterThuillier, López-Pastor y Fuentes (2017) indican que, a pesar del aumento importante de las publicaciones sobre inmigración, deporte y escuela en los últimos años, es un campo de estudios que necesita ser explorado con mayor profundidad. Por ello, nos gustaría animar a que más docentes de $\mathrm{EF}$ incorporen propuestas innovadoras relacionadas con la educación intercultural similares a la expuesta en este trabajo, con el fin de poder comparar experiencias similares en un futuro.

\section{Agradecimientos}

Agradecemos su colaboración a todos los voluntarios de la asociación ESN y a los estudiantes erasmus que participaron en la innovación 
educativa, ya que sin ellos la innovación educativa Erasmus in Schools no hubiese sido posible.

\section{Referencias}

Aguado, M. T. (1996). Atención a la diversidad cultural e igualdad escolar. Modelo para el diagnóstico y desarrollo de actuaciones educativas en contextos escolares multiculturales. Madrid: CIDE.

Alonso, J. A., Cachón, J., Castro, R., \& Zagalaz, M. L. (2015). Propuesta didáctica bilingüe para educación física en educación primaria. Juegos populares tradicionales ingleses. Retos: Nuevas tendencias en Educación Física, Deporte y Recreación, 28, 116-121.

Bartolomé, M. (2001). Identidad ciudadana en adolescentes. Nuevos enfoques desde la educación intercultural. En Soriano, E. (coord.), Identidad cultural y ciudadanía intercultural (pp. 75-110). Madrid: La Muralla.

Brown, S. (2001). Does high school racial composition matter? The effect of race and racial composition on high school students' percepctions and sucess. Michigan, MI: Ann Arbor.

Carbonell, J. (2006). La aventura de innovar. El cambio en la escuela. Madrid: Morata.

Carter-Thuillier, B., López-Pastor, V. M., \& Fuentes, F. (2017). Inmigración, deporte y escuela. Revisión del estado de la cuestión. Retos: Nuevas tendencias en Educación Física, Deporte y Recreación, 32, 19-24.

Cifuentes, A., Torrego, L., \& Siles, G. (2012). Presencia del streaming, mixture einclusion en los centros educativos españoles de primaria y secundaria. Resultados de la encuesta nacional del proyecto I+D, MIXSTRIN. Revista Interuniversitaria de Formación del Profesorado, 26, 89-104.

Comisión Europea (2006, 8 de septiembre). Eficiencia yequidad en los sistemas europeos de educación y formación. Bruselas: Comunicación de la Comisión al Consejo y al Parlamento Europeo. Recuperado de http://eurored.ccoo.es/comunes/recursos/99999/

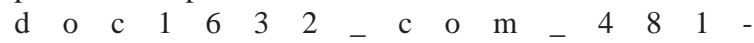

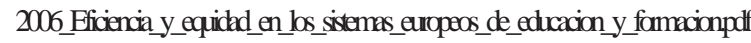

De Botton, L., Flecha, F., \& Puigvert, L. (2009). El éxito escolar no depende de la proporción de inmigrantes sino de la aplicación de las actuaciones de éxito. Revista de la Asociación de Sociología de la Educación (RASE), 2(3), 45-55.

Derri, V., Kellis, I., Vernadakis, N., Albanidis, E., \& Kioumourtzoglou, E. (2014). The effect of an intercultural Physical Education Program in comparison to the typical one on students' social skills learning. Journal of Human Sport and Exercise, 9(1), 91-102.

Duque, E., Holland, C., \& Rodríguez, J. (2012). Mixture, streaming e inclusion: tres formas de agrupar al alumnado. Cuadernos de Pedagogía. 429, 28-30.

Fernández, M. (2001). La educación intercultural en la sociedad multicultural. Revista de Organización y Gestión Educativa, 6, 37.

Fernández, M. (2003). La segunda generación ya está aquí. Papeles de Economía Española, 98, 238-261.

Flecha, R., \& Puigvert, L. (2002). Multiculturalismo y Educación. En T. Lleixà (Ed.), Multiculturalismo y Educación Física (pp. 9-45). Barcelona: Paidotribo.

Flecha, R., \& Puigvert, L. (2011). Contra el racismo. Acciones e investigaciones sociales, 11, 135-164.

Flores, G. (2013). El profesorado de educación física ante la realidad multicultural: percepción sobre el alumnado, intervención pedagógica y trayectoria profesional. Un estudio de casos. Tesis Doctoral de la UniversidadAutónoma de Barcelona, Bellaterra. Recuperado de http://ddd.uab.cat/record/117882

Flores, G., Prat, M., \& Soler, S. (2014). La voz del profesorado de educación física sobre su formación académica ante la realidad multicultural: análisis de la situación y propuestas de mejora. Revista Electrónica Interuniversitaria de Formación del Profesorado, 17(2), 183-199.
Flores, G, Prat, M., \& Soler, S. (2015a). Echando la vista atrás: la voz del profesorado de educación física sobre su trayectoria profesional en una escuela multicultural. Apunts. Educación Físicay Deportes, 122, 88-98.

Flores, G., Prat, M., \& Soler, S. (2015b). La intervención pedagógica del profesorado de educación física en un contexto multicultural: prácticas, reflexiones y orientaciones. Retos: Nuevas tendencias en Educación Física, Deporte y Recreación, 28, 248-255.

Flores, G, Prat, M., \& Soler, S. (2017). La visión del profesorado de Educación Física sobre la presencia del alumnado de origen extranjero en la escuela: ¿oportunidad o problema? Retos: Nuevas tendencias en Educación Física, Deporte y Recreación, 31, 64-68.

García, F., Rubio, M., \& Bouachra, O. (2008). Población inmigrante y escuela en España: un balance de investigación. Revista de Educación, 345, 23-60.

García-Fernández, J. A., \& Goenechea, C. (2009). Educación intercultural: análisis de la situación y propuestas de mejora. Barcelona: Wolters Kluwer España.

Hildebrandt-Stramann, R. (2016). Integração/inclusão por meio de relações de movimento. Ágora para la Educación Física y el Deporte, 18(3), 214-229.

Imbernon, F. (1996). En busca del discurso educativo: la escuela, la innovación educativa, el currículum, el maestro y su formación. Buenos Aires: Magisterio del Río de la Plata.

López-Pastor, V. M., Pérez, Á., \& Monjas, R. (2007). La atención a la diversidad en el área de educación física. La integración del alumnado con necesidades educativas específicas, especialmente el alumnado inmigrante y de minorías étnicas. Lecturas: Educación Física y Deportes, 106. http://www.efdeportes.com/efd106/la-atencion-ala-diversidad-en-educacion-fisica.htm

Martos, D. (2009). Jugar a la pelota valenciana en silla de ruedas. Tándem. Didáctica de la Educación Física, 31, 101-110.

Maza, G. (2002). El deporte y su papel en los mecanismos de reproducción social de la inmigración inmigrada extranjera. Apunts. Educación física y deportes, 68, 58-66.

Medina, F. X. (2002). Deporte, inmigración e interculturalidad. Apunts. Educación Física y Deportes, 68, 18-23.

Ortiz, M. (2014). Inmigración, escuela y exclusión. EMPIRIA. Revista de Metodología de las Ciencias Sociales, 28, 59-78.

Rangvid, B.S. (2005). Sources of immigrants' Underachievemen: results from m PISA-Copenhagen. New York: Columbia University Press.

Ruiz, M., Molero, D., Zagalaz, M. L., \& Cachón, J. (2012). Análisis de la integración del alumnado inmigrante a través de las clases de Educación Física. Apunts. Educación Física y Deportes, 108, 2634.

Sáez,A. (2006). La educación intercultural. Revista de Educación, 339, 859-881.

Soler, S., Flores, G., \& Prat, M. (2012). La educación física y el deporte como herramientas de inclusión de la población inmigrante en Cataluña: el papel de la escuela y la administración local. Pensar a Prática, 15(1), 1-19.

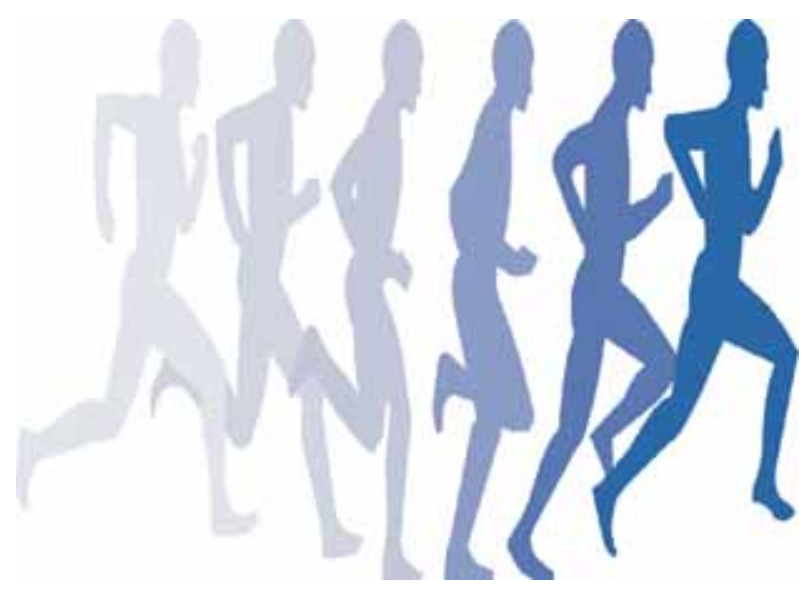

